\title{
An Intelligent Agent based Land Encroachment Detection Approach
}

\author{
Simon Dacey, Lei Song, and Shaoning Pang \\ Department of Computing, Unitec Institute of Technology, Private Bag 92035, \\ Auckland, New Zealand
}

\begin{abstract}
Land management and planning is essential to assist the economic growth, sustainable resource use and environmental protection of a city. This paper describes a novel approach to automatic encroachment detection to assist in land management decision making. The approach begins with training the agent to identify and understand the land cover/use features (such as buildings, parks, trees and roads) that are predominant in the region of interest, and carries out segmentation on the park data using the intelligent agent developed from the training samples. Experiments on park images from Auckland New Zealand show the effectiveness of the proposed approach.
\end{abstract}

\section{Introduction}

The use of public land for private purposes has been identified as a problem affecting public parks in the Auckland region. Dacey et al. [1] proposed a participatory approach to the problem of encroachment. This approach involves visiting each park with GPS equipment to obtain data on the actual physical boundary. The data collection proved to be very time-consuming so a quicker way of identifying parks with likely encroachment is needed.

Land encroachment is defined as the change in the perceived or actual use of land from either (a) human caused encroachment: the use of public land for private purposes, or (b) environmental caused encroachment: the change in the ability of land for its original purpose from an environmental change. This paper examines the use of digital image analysis in automatically detecting encroachment on public parks in two specific areas in Auckland, New Zealand.

When dealing with encroachment it is necessary to differentiate between land cover change and land use change. For example if an area of grassland belonging to a public park has been fenced off by the resident of a property that is adjacent to the park, the land cover of the majority of the grassland has not changed but its land use has changed from public to private. Detection of this type of change requires the use of high resolution data as features such as fences are hard to detect and/or difficult to distinguish from features, such as paths, that are not indicative of encroachment.

In practice, there are 4 general types of encroachment studied:

1 Permanent land cover/use change. For example a building has been built either wholly on the park or partially on it. 
2 Permanent land use change only. For example Public Park area has been fenced off so is no longer accessible to public but land cover has not changed (i.e. land cover is still grass).

3 Temporary land cover/use change. For example the park (or part of the park) has been used as a garbage dumping site in a way such that it can no longer be used by the public as a park.

4 Physical boundary concealed by land cover. For example a boundary fence has been moved, or removed completely, in an area that is obscured by dense vegetation.

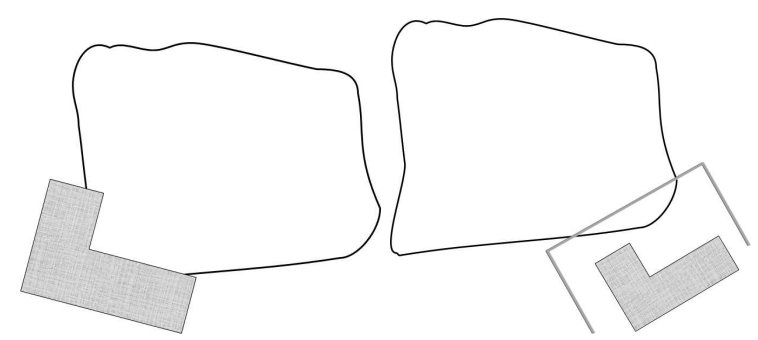

(a)

(b)

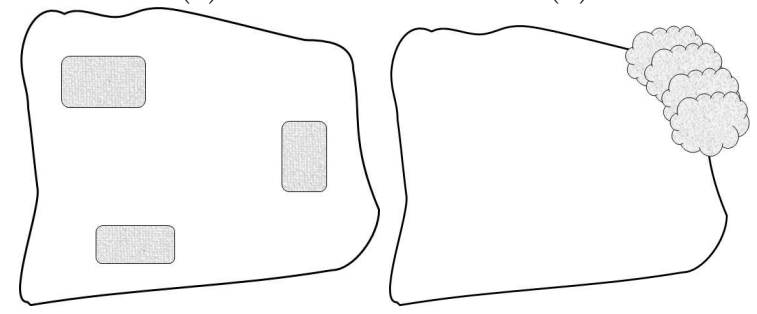

(c)

(d)

Fig. 1: The four types of encroachment. (a) Permanent land cover/use change; (b) Permanent land use change only; (c) Temporary land cover/use change; and (d)Physical boundary concealed by land cover.

A new approach is in demand because the existing methods are unsuitable for our specific needs, and the applications examined are not reproducible in the New Zealand context. The strengths of the proposed method is that it can detect the types of land encroachment identified above. Additionally, it is suited to the type of land cover (urban and rural mixed) found in Auckland, as we design, build and test a solution for our specific purpose. Although trees naturally form part of a public park, they need to be differentiated because they can blur the boundary of a park and can obscure encroachment. 


\section{Methodology}

In this section, we propose a new agent-based image solution to land-encroachment detection. As seen in the literature, agent-based image analysis has been used in a variety of applications such as range-image segmentation [2], and off-road vehicle guidance, [3]. The advantage offered by such a solution includes: (1) image processing parallelization; and (2) the flexibility to concentrate on either spatial or temporal changes. A review of agent-based image segmentation can be found in [4].

The flowchart in Fig. 2 shows an outline of the proposed land encroachment detection approach. The aim of the approach is to detect any changes between a pair of multi-temporal images, which we identify as source image $I_{0}$ and target image $I_{1}$ in this paper. The principle of the approach is that the more that an agent learns about $I_{0}$, the more accurate any change detection (against $I_{0}$ ) will be.

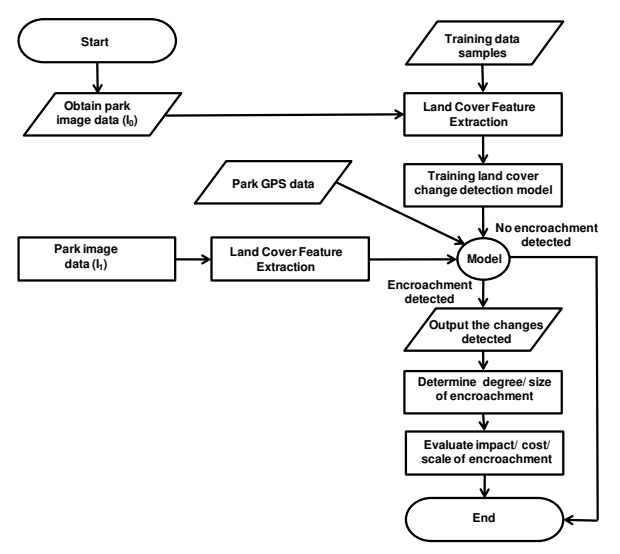

Fig. 2: Flowchart of proposed approach

To learn as much prior knowledge as possible, about $I_{0}$, in terms of the type of land cover, additional image data is collected for training the agent with the knowledge of 5 land cover patterns such as Grass, Buildings, Trees, Fences and Roads, which represent the land cover/use characteristics that are most likely to be found in or around public parks.

The agent with the prior knowledge of basic land cover is expected to learn the source image $I_{0}$ incrementally (i.e. incremental learning). When performing land encroachment detection, the target image $I_{1}$ is processed by the agent to detect the change against $I_{0}$. If encroachment has been detected then the size of the encroachment can be calculated. Then the risk factor of the encroachment is examined using the size of the encroachment and the rateable value of the land that has been encroached upon.

Details on how the approach is carried out are given next. 


\subsection{Land Cover Feature Extraction}

The land cover of a public park is usually grassland. However, in some cases, the types of land cover in a public park can include buildings, play grounds, paths and roads. The automatic detection of encroachment has to be able to take spatial co-ordinates into consideration.

For better understanding of the image characteristics, we apply first an image processing method - Gabor filter - to enhance images characteristics, such as texture and contour information. A general Gabor filter function is expressed as

$$
G(x, y ; \lambda, \theta, \psi, \sigma)=\exp \left(-\frac{x^{\prime}+y^{\prime 2}}{2 \sigma^{2}}\right) \exp \left(x\left(2 \pi \frac{x^{\prime}}{\lambda}+\psi\right)\right),
$$

where

$$
\left[\begin{array}{l}
x^{\prime} \\
y^{\prime}
\end{array}\right]=\left[\begin{array}{cc}
\cos (\theta) & \sin (\theta) \\
-\sin (\theta) & \cos (\theta)
\end{array}\right]\left[\begin{array}{l}
x \\
y
\end{array}\right],
$$

and $\lambda$ represents the sinusoid's wavelength, $\theta$ represents the orientation, $\psi$ is the phase offset, and $\sigma$ denotes the spread of the Gaussian window [5].

Given a set of orientations $\theta^{t}$, where $t=1,2, \ldots, T$, and according to (1) and (2), using an image $I$ as input, we obtain $T$ different images $G_{\theta^{t}}^{I}$, which are the data representatives of these Gabor filtered images in $T$ orientations. For example, let the 2-D image $I$ with a size of $M \times N$ be processed by Gabor filter, and the magnitude of complex-valued subbands is denoted as

$$
G_{\theta^{t}}^{I}=\left\{m_{\theta^{t}}^{I}(m, n) \mid 1 \leq m \leq M, 1 \leq n \leq N, \text { and } t=[1,2, \ldots, T]\right\},
$$

where $G_{\theta^{t}}^{I}$ is a set of real-valued 2-D subbands signals, representing the magnitude of complex-valued wavelet coefficients obtained at the direction $\theta^{t}$. The magnitudes of each directional subband's coefficients are computed and collectively denoted by $G_{\theta^{t}}^{I_{0}}$ and $G_{\theta^{t}}^{I_{1}}$ for the multiple temporal images $I_{0}$ and $I_{1}$.

\subsection{Encroachment Detection by Land Cover Change Detection}

Knowledge discovery from the source image $\boldsymbol{I}_{\mathbf{0}}$. In order to learn content composition of the collected data $D_{0}$ for $I_{0}$, we employ a clustering algorithm to discriminate different image contents from each other and group similar contents together. To maximize the flexibility of our method, the general mathematic definition of clustering is shown below.

Given $D_{0}=\left\{D_{0}(r) \mid 1 \leq r \leq R\right\}, \boldsymbol{C}=\left\{\boldsymbol{c}_{\boldsymbol{k}} \mid 1 \leq k \leq K\right\}$ denote the set of $K$ clusters' core vectors, and $\Re_{k}$ represents the number of vectors assigned to $k$-th cluster, initially, $k=1$ and $\boldsymbol{c}_{\boldsymbol{k}}=D_{0}(1)$. Normally, clustering is to assign data that are more "alike" into one cluster [6]. To determine the word "alike", a function for measuring similarity among data can be formulated as (2.2),

$$
\text { Similarity }(\boldsymbol{a}, \boldsymbol{b})=\left\{\begin{array}{l}
0 \text { if two vectors } \boldsymbol{a} \text { and } \boldsymbol{b} \text { are similar } \\
1 \text { otherwise }
\end{array}\right.
$$


According to (2.2), we compare the similarity between $D_{0}$ and $C$, for each $D_{0}(r)$ and $\boldsymbol{c}_{\boldsymbol{k}}$, where $r=1,2, \ldots, R$ and $k=1,2, \ldots, K$.

if Similarity $\left(D_{0}(r), \boldsymbol{c}_{\boldsymbol{k}}\right)=0$, assign $D_{0}(r)$ to $k$ th cluster, and let $c_{k}=$ $\frac{\Re_{k} \boldsymbol{c}_{\boldsymbol{k}}+D_{0}(r)}{R_{k}+1}$, otherwise Similarity $\left(D_{0}(r), \boldsymbol{c}_{\boldsymbol{k}}\right)=1$, then assign $D_{0}(r)$ to $(k+1)$ th cluster, and let $\boldsymbol{c}_{\boldsymbol{k}+\boldsymbol{1}}=D_{0}(r)$. Hereafter, let $r=1$ we can repeat this process until the last input vector $D_{0}(R)$ has been assigned into one cluster. At the end of clustering, the cluster labels $Y_{0}=\{1,2, \ldots, K\}$ and $D_{0}=\left[D_{0}, Y_{0}\right]$ can be obtained.

As mentioned above, this stage is flexible via selecting various clustering algorithms, since there exist a variety of clustering techniques. In this research, $\mathrm{K}$-mean clustering, as the first choice, is utilized.

For the purpose of storing $I_{0}$ as small size knowledge instead of keeping the same size of $I_{0}$, machine learning model is employed.

Weighted incremental linear proximal support vector machine (wIncLPSVM) is designed for solving class-imbalance problem. It is suitable for our study because of its following properties: 1) it is an incremental learning algorithm capable of learning a huge size input image piece by piece; 2 ) in contrast to the traditional incremental LPSVM, the samples from positive and negative are processed separately in wIncLPSVM which makes it easy to observe the effect of one step incremental learning.

The core model used in this work is a matrix component in wIncLPSVM model namely $\boldsymbol{M}$ which condenses the knowledge of positive class. In practice, we train a $\boldsymbol{M}_{\mathbf{0}}{ }^{O_{k}}$ for each object obtained from the objects decomposition on image $I_{0}\left(D_{0}^{\prime}\right)$. For the $k$ th object in $I_{0}$, the model is calculated as

$$
\boldsymbol{M}_{0}^{O_{k}}=\left[\boldsymbol{D}_{0}^{O_{k}}-\boldsymbol{e}\right]^{T}\left[\boldsymbol{D}_{0}^{O_{k}}-\boldsymbol{e}\right],
$$

where $\boldsymbol{e}=[1, \ldots, 1]^{T}$. After the training phase, we conduct one step incremental learning to update $\boldsymbol{M}_{0}^{O_{k}}$

$$
\boldsymbol{M}_{0}^{O_{k}}(r)=\boldsymbol{M}_{0}^{O_{k}}+\left[\boldsymbol{D}_{\mathbf{0}}^{\boldsymbol{O}_{k}}(r)-\boldsymbol{e}\right]^{T}\left[\boldsymbol{D}_{\mathbf{0}}^{\boldsymbol{O}_{\boldsymbol{k}}}(r)-\boldsymbol{e}\right],
$$

using the each sample from data $D_{0}$ and use the eigenvalue of updated $\boldsymbol{M}_{0}^{O_{k}}$ to measure the influence of incoming sample. By going through all samples in each object on corresponding model, a range of influences can be obtained for each object. A range Range $_{0}^{O_{k}}$ is a closed interval defined by the maximal and minimal eigenvalues as

$$
\text { Range }_{0}^{O_{k}}=\left[\min \left(\operatorname{eig}\left(\boldsymbol{M}_{0}^{O_{k}}\right)\right), \max \left(\operatorname{eig}\left(\boldsymbol{M}_{0}^{O_{k}}\right)\right)\right] .
$$

The incremental learning is conducted on learning the model $\boldsymbol{M}_{0}^{O_{k}}$, so the image $I_{0}$ can be learned piece by piece. For example, $D_{0}$ is separated into $N$ pieces with 5 objects. There are five $\boldsymbol{M}_{0}^{O_{k}}$ models, and each of them can be stored in a $20 \times 20$ matrix. In contrast, $D_{0}$ is a $154814 \times 20$ matrix, the stored model $\left\{\boldsymbol{M}_{0}^{O_{k}} \mid k \in[1,5]\right\}$ includes five $20 \times 20$ matrices. Thus the size of stored knowledge is 154 times smaller than original image dataset $D_{0}$. 


\subsection{Change Detection on the target image $I_{1}$}

One-step-more incremental learning detect changes against $I_{0}$ by measuring the effect of incremental learning on new incoming data $I_{1}$. Usually, based on the model of batch learning, incremental learning is conducted continuously step by step from the appearance of the first sample to the completion of that all samples being learned. However, in our method, while a new sample appears, the incremental learning is merely conducted once (i.e., one step) on the basic model obtained from $I_{0}$. Hereafter, when the next sample comes, the incremental learning agent starts over from the original $I_{0}$ base model and repeat the incremental learning detection step. In other words, while conducting image change detection the incremental learning agent collects no new knowledge in memory.

In the proposed approach, as a result of knowledge discovery on $I_{0}, I_{0}$ now is represented as an incremental learning model $\boldsymbol{M}_{\mathbf{0}}$.

$$
\boldsymbol{M}_{\mathbf{0}}=\left\{<\boldsymbol{M}_{0}^{O_{k}}, \text { Range }_{0}^{O_{k}}>\right\}, k=1, \ldots, K
$$

The basic detection model $\boldsymbol{M}_{0}^{O_{k}}$ is formulated by (5). The range $\operatorname{Range}_{0}^{O_{k}}$ is given by one-step learning (6) and (7). Lets assume that each data sample from $D_{1}$ (the collected data from $I_{1}$ ) has the same object label as its corresponding sample from $D_{0}$. The one-step more incremental learning on $I_{1}$ can be conducted on each object of $I_{0}$ by (9) as,

$$
\boldsymbol{M}_{1}^{O_{k}}(r)=\boldsymbol{M}_{0}^{O_{k}}+\left[\boldsymbol{D}_{1}^{\boldsymbol{O}_{k}}(r)-\boldsymbol{e}\right]^{T}\left[\boldsymbol{D}_{1}^{\boldsymbol{O}_{k}}(r)-\boldsymbol{e}\right] .
$$

The effect of new sample $\boldsymbol{D}_{\mathbf{1}}^{\boldsymbol{O}_{\boldsymbol{k}}}(r)$ to $\boldsymbol{M}_{0}^{O_{k}}$ can be calculated via (10).

$$
\text { effects }(r)=\operatorname{eig}\left(M_{1}^{, O_{k}}(r)\right) \text {. }
$$

Then, the change status of the $r$-th sample is determined by $\operatorname{effects}(r)$ following the rule as,

$$
\text { Change }(r)=\left\{\begin{array}{l}
0, \text { effects }(r) \in \text { Range }_{0}^{O_{k}} \\
1, \text { effects }(r) \notin \text { Range }_{0}^{O_{k}}
\end{array}\right.
$$

Consequently, a binary image (or mask) $B_{O_{k}}=\left\{b_{o_{k}}(m, n) \mid 1 \leq m \leq M, 1 \leq n \leq N\right\}$ are formed for each object defined in $I_{0}$.

\section{Experiments and Discussions}

A number of experiments were conducted to study the effectiveness of the new approach. In this section we look at the data used in the experiments, the setup and procedure of the experiments and we discuss the results of the experiments.

The data used in the experiments is digital image data of New Zealand specifically the Auckland area. Auckland City Council covers the entire Auckland region in which there are over 800 designated public parks and reserves to examine for possible encroachment. The range in the parks' size is substantial, for example in the Manukau area the parks range in size from 38 square meters to 1995880 square meters. The data sets used for the detection of encroachment are digital images of areas that include public parks. 
1 Aerial Photographs. The aerial photographs are captured on-line. The scale is set manually to $7.5 \mathrm{~cm} / 234 \mathrm{~m}$ at time of capture and the ratio is one pixel to 0.5 meters. The images are in JPEG format and are collected from Google Earth.

2 Land Boundary Data. The boundary data is used to verify that encroachment has been detected and to estimate the size of encroachment. When this research was started, there were seven Auckland regional councils and of those councils approached 2 of them, Manukau and North Shore supplied boundary data in GIS shape file format.

To demonstrate the automatic encroachment detection technique, for this paper, four parks: Wyllie Park, Teviot Reserve, Auburn Reserve, and Diana Reserve are selected as representative examples of the four categories of park discussed above.

The experiments were carried in three phases. The first phase involves teaching the agent prior knowledge about the land cover patterns such as grass, buildings, trees, fences and roads, as they are most commonly found in and around public parks in Auckland. Note that, for this purpose, we collect data only from non-park areas for the training of the agent. After the first phase learning, the agent now has the prior knowledge (i.e., the number of clusters $k$ and initial mean vector of each cluster). In the second phase, we set the equipped agent to perform spatial incremental learning on $I_{0}$ in order to obtain knowledge from the source image. The third phase is change detection on $I_{1}$. We set the $I_{0}$ learned agent to repeat the incremental learning process on $I_{1}$ to detect change of $I_{1}$ against $I_{0}$.

\subsection{Land Cover Change Detection Results}

Four specific experiments are carried out:

1 To detect permanent encroachment such as buildings.

2 To detect encroachment in the form of areas of a park fenced off.

3 To detect temporary encroachment such as vegetable-growing.

4 To detect possible areas of encroachment that may obscured by natural objects such as trees.

Permanent Land Cover Encroachment Type 1: Permanent land cover encroachment is where a building is constructed illegally on a public park. In land management terms this is the most serious form of encroachment. Although it is relatively simple to detect the presence of a building on a park, one difficulty in detecting permanent encroachment is the misclassification of legitimate constructions. One solution to this problem is to capture multiple images over an extended time-frame and make continuous comparisons. The type of structure built can range from a movable private structure (e.g. a shed), an immovable private structure (e.g. a deck) through to commercial buildings and government building 
Fig. 3(a) shows Wyllie Park with the council boundary, Wyllie Park with a house encroaching near the top-left corner and the results of the image segmentation algorithm: white pixels indicate encroachment. In the next section we detail the performance of our method of classification.
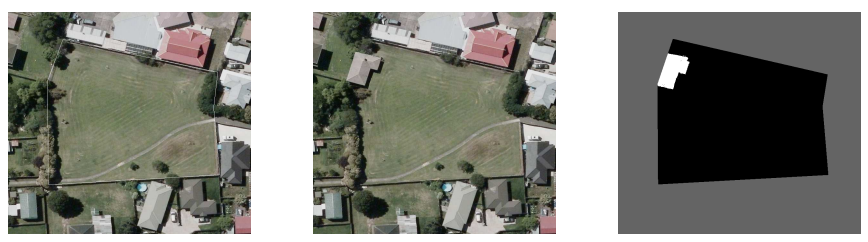

(a) Wyllie park
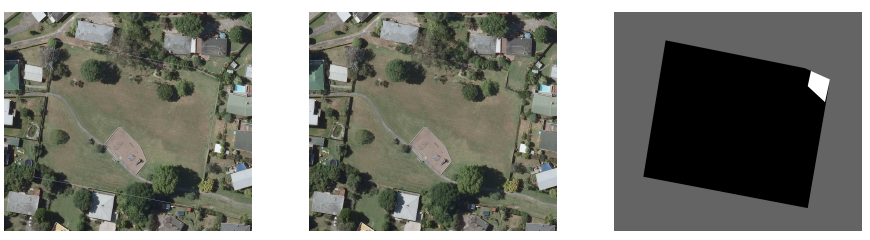

(b) Teviot reserve
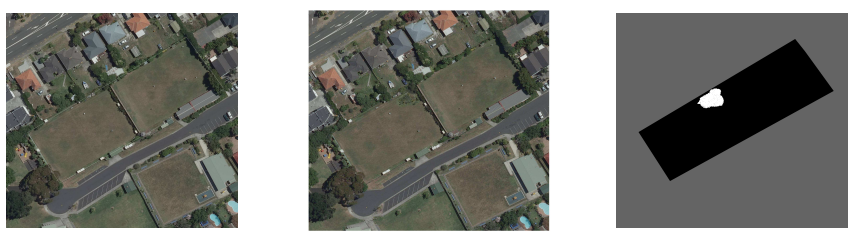

(c) Auburn reserve
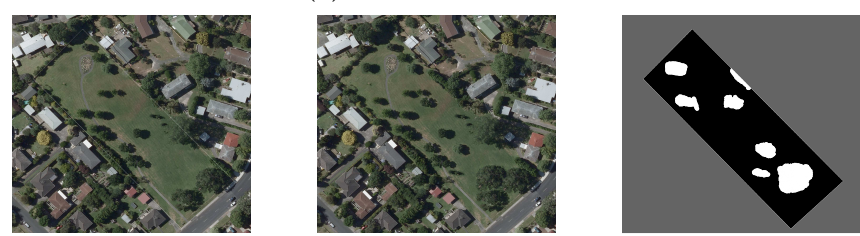

(d) Diana reserve

Fig. 3: Experiment results

Type 2: We provide a permanent land use encroachment example where a fence is built illegally on a park, see Fig 5. In land management terms this is the another serious form of encroachment. This type of encroachment can be one of the most difficult to detect because the land cover change is usually not as noticeable as that of a building. This is especially true when the fence or enclosure structure is only a few pixels thick in the observed image. Contrariwise, when 
these enclosure boundaries increase in pixel width they become much easier to detect.

Fig. 3(b) shows Teviot Reserve with the council boundary, the ground truth image with an introduced fence element and the results of the image segmentation approach, white pixels classify encroachment.

Type 3: Temporary Use Encroachment, for example vegetable-growing, is not as serious as permanent encroachment as it is normally easier to resolve by the authorities. This type of encroachment can be difficult to detect especially when the colour of the vegetation is similar to the naturally occurring colours in the park. Another example of this type of encroachment is the detection of illegal garbage disposal on public land, this problem is usually easier to detect as the garbage colours usually differ in contrast and hue from the background. Temporary encroachment could become permanent if it is not dealt with by the authorities in a timely manner. Other types of temporary encroachment include organic dumping (e.g. grass cuttings) and inorganic dumping (e.g. furniture), grazing (e.g. horses) and intrinsic value (e.g. a fruit tree planted).

Fig. 3(c) shows Auburn Reserve with the council boundary shown on an overlay, the ground truth image with an introduced vegetation element and the results of the image segmentation approach, white pixels indicate the vegetation encroachment detection.

Type 4: Physical boundary encroachment is a difficult problem to solve, especially when the boundary encroachment is concealed under a canopy of vegetation. However, not all vegetation canopies will hide illegal encroachment.

Fig. 3(d) shows the Diana Reserve park area, the ground truth image with an introduced plant elements and the results of the image segmentation approach, white pixels show the areas of newly introduced plant life. In many instances, this plant life will be introduced by the authorities, especially when it is in the confines of the park. However, the elements detected on park boundaries should indicate a possible encroachment, as these elements may have been illegally placed or provide canopies for illegal encroachment. These type of boundary triggers should warrant further investigation by the authorities.

\begin{tabular}{ccccccc}
\hline Park & Encroachment & Park Size & Encroachment Size & Encroachment & \multicolumn{2}{c}{ Park Rateable } \\
Name & Type & (pixels) & (pixels) & Scale & Value(NZD) & Value(NZD) \\
\hline Wyllie & Building & 230,214 & 7,442 & 0.03 & $485,000.00$ & $14,550.00$ \\
Teviot & Area of park fenced off & 2007,890 & 158,297 & 0.07 & $510,000.00$ & $35,700.00$ \\
Auburn & Introduced vegetation & 735,456 & 20,284 & 0.027 & $2150,000.00$ & $59,288.00$ \\
Diana Newly introduced plant life & 1260,279 & 154,431 & 0.12 & $690,000.00$ & $84,550.00$ \\
\hline \multicolumn{2}{l}{ Table } \\
\hline
\end{tabular}

Table 1: Details of the encroachment detected on the chosen parks

Table 1 above shows the experiment results in the terms of the size and scale of the encroachment for each of the parks. The rateable value of each park is obtained from the Auckland City Council [7]. The size of encroachment in pixels is divided by the total area of the park in pixels to give the scale of encroachment. The rateable value of the park is multiplied by the scale of encroachment to give the commercial value of the encroachment. As stated in 
the introduction there are four main types of encroachment and each of the four parks used in the experiments is an example of one of the four types. The impact of the encroachment depends on the type of encroachment and the scale of encroachment.

\section{Conclusions and Future Work}

The results of the experiments show that the approach is successful in highlighting possible occurrences of encroachment. The the main disadvantage of the approach is that cannot work as a stand-alone solution - ground survey data collection needs to take place to confirm the occurrence of encroachment. The advantages of the approach are that permanent encroachment (such as buildings and fences) can be detected and temporary encroachment (such as vegetablegrowing and garbage dumping) can also be detected. Another disadvantage of the approach is that it is not as reliable in detecting encroachment and/or changes in areas where there is a high concentration of tree as these area have a high variation in colors and concentration

\section{References}

1. Dacey, S. and Barbour, R.H. and Fernando, A.: Participatory Land Use Management of Public Spaces in New Zealand. In: Proceedings of the JURSE conference on urban remote sensing and planning, pp. 449-452. Munich, Germany (2011)

2. Mazouzi, S., Guessoum, Z., and Michel, F., Batouche, M.: An Agent-Based Approach for Range Image Segmentation. In: Jamali, N., Scerri, P., Sugawara, T. (eds.) Massively Multi-Agent Technology 2008. pp. 146-161. Springer, Heidelberg (2008)

3. Broggi, A., Cattani, S.: An agent based evolutionary approach to path detection for off-road vehicle guidance. Pattern Recogn. Lett. 27(11) 1164-1173 (2006)

4. Mishra, P., Srivastava, N., Shukla, K.K., Singhal, A.: Agent based Image Segmentation Methods: A Review. Interational Journal Comp. Tech. Appl. 2(3), 704-708 (2011)

5. Daugman, J.G.: Uncertainty relation for resolution in space, spatial frequency, and orientation optimized by two-dimensional visual cortical filters. Journal of the Optical Society of America A Optics and image science 2,7, 1160-1169 (1985)

6. Huang, Z.: Extensions to the k-Means Algorithm for Clustering Large Data Sets with Categorical Values. Data Mining and Knowledge Discovery 2(3) 283-304 (1998)

7. Auckland City Council: Auckland GIS Viewer, http://maps.aucklandcouncil. govt.nz/aucklandcouncilviewer/ 